\title{
Synthesis, Crystal Structure \\ and Thermodynamic Properties \\ of Apatite-like Lead Gadolinium Vanadato-germanates
}

\author{
Lyubov T. Denisova*a, Maksim S. Molokeev ${ }^{\mathrm{a}, \mathrm{b}}$, \\ Evgenia O. Golubeva a and Nadezhda A. Galiakhmetova ${ }^{a}$ \\ ${ }^{a}$ Siberian Federal University \\ Krasnoyarsk, Russian Federation \\ ${ }^{b}$ Kirensky Institute of Physics SB RAS \\ Krasnoyarsk, Russian Federation
}

Received 29.11.2021, received in revised form 01.02.2022, accepted 04.02.2022

\begin{abstract}
The $\mathrm{Pb}_{10-\mathrm{x}} \mathrm{Gd}_{\mathrm{x}}\left(\mathrm{GeO}_{4}\right)_{2+\mathrm{x}}\left(\mathrm{VO}_{4}\right)_{4-\mathrm{x}}(\mathrm{x}=0.5,1.0)$ apatites were synthesized by the solid-phase synthesis by roasting stoichiometric mixtures of $\mathrm{PbO}, \mathrm{Gd}_{2} \mathrm{O}_{3}, \mathrm{GeO}_{2}$, and $\mathrm{V}_{2} \mathrm{O}_{5}$ in air at temperatures of 773-1073 K. Their crystal structure was determined using X-ray diffraction analysis. The high-temperature heat capacity $(350-1000 \mathrm{~K})$ was measured by differential scanning calorimetry. The experimental data $\mathrm{Cp}=\mathrm{f}(\mathrm{T})$ were used to calculate the thermodynamic properties of apatites.
\end{abstract}

Keywords: apatites, solid-phase synthesis, crystal structure, high-temperature heat capacity, thermodynamic properties.

Acknowledgements. The work is performed as a part of the state assignment for the science of Siberian Federal University, project number FSRZ-2020-0013. We are grateful to the Krasnoyarsk Regional Center for Collective Use, Krasnoyarsk Scientific Center, Siberian Branch, Russian Academy of Sciences.

Citation: Denisova, L. T., Molokeev, M. S., Golubeva, E. O. Galiakhmetova, N. A. Synthesis, crystal structure and thermodynamic properties of apatite-like lead gadolinium vanadato-germanates. J. Sib. Fed. Univ. Chem., 2022, 15(1), 128-136. DOI: 10.17516/19982836-0277

(C) Siberian Federal University. All rights reserved

This work is licensed under a Creative Commons Attribution-NonCommercial 4.0 International License (CC BY-NC 4.0).

* Corresponding author E-mail address: ldenisova@sfu-kras.ru 


\title{
Синтез, кристаллическая структура
}

\section{и термодинамические свойства}

апатитоподобных ванадато-германатов

свинца гадолиния

\author{
Л. Т. Денисова ${ }^{a}$, М. С. Молокеев ${ }^{\mathrm{a}, \boldsymbol{\sigma}}$, \\ Е.О. Голубева ${ }^{a}$, Н.А. Галиахметова ${ }^{a}$ \\ ${ }^{a}$ Сибирский федеральный университет \\ Российская Федераџия, Красноярск \\ ${ }^{6}$ Институт физики им. Л. В. Киренского \\ ФИЦ КНЦ СО РАН \\ Российская Федераџия, Красноярск
}

Аннотация. Методом твердофазных реакций обжигом на воздухе стехиометрических смесей $\mathrm{PbO}, \mathrm{Gd}_{2} \mathrm{O}_{3}, \mathrm{GeO}_{2}$ и $\mathrm{V}_{2} \mathrm{O}_{5}$ в интервале температур 773-1073 K синтезированы апатиты $\mathrm{Pb}_{10-\mathrm{x}} \mathrm{Gd}_{\mathrm{x}}\left(\mathrm{GeO}_{4}\right)_{2+\mathrm{x}}\left(\mathrm{VO}_{4}\right)_{4-\mathrm{x}}(\mathrm{x}=0.5,1.0)$. С использованием рентгеноструктурного анализа определена их кристаллическая структура. Методом дифференциальной сканирующей калориметрии измерена высокотемпературная теплоемкость (350-1000 К). По экспериментальным данным $\mathrm{C}_{\mathrm{p}}=\mathrm{f}(\mathrm{T})$ рассчитаны термодинамические свойства апатитов.

Ключевые слова: апатиты, твердофазный синтез, кристаллическая структура, высокотемпературная теплоемкость, термодинамические свойства.

Благодарности. Авторы выражают благодарность Красноярскому региональному центру коллективного пользования ФИЦ КНЦ СО РАН. Работа выполнена при частичной финансовой поддержке в рамках государственного задания на науку ФГАОУ ВО «Сибирский федеральный университет», номер проекта FSRZ-2020-0013.

Цитирование: Денисова, Л.Т. Синтез, кристаллическая структура и термодинамические свойства апатитоподобных ванадато-германатов свинца гадолиния / Л. Т. Денисова, М.С. Молокеев, Е. О. Голубева, Н. А. Галиахметова // Журн. Сиб. федер. ун-та. Химия, 2022, 15(1). С. 128-136. DOI: 10.17516/1998-2836-0277

\section{Введение}

Апатиты, обладая уникальными физико-химическими свойствами, уже в течение длительного времени привлекают к себе внимание исследователей и практиков [1-10]. Они используются в качестве биоматериалов, лазерных, люминесцентных и оптических материалов $[3,11]$. Важной особенностью апатитов является способность их структурных единиц к замещению другими ионами без существенного изменения структуры. При этом свойства таких соединений, как правило, изменяются. Поэтому изоморфное замещение является одним из подходов, используемых для создания новых материалов. Так, например, авторы ра- 
боты [12], замещая часть свинца в апатите $\mathrm{Pb}_{5}\left(\mathrm{GeO}_{4}\right)\left(\mathrm{VO}_{4}\right)_{2}$ на лантан, получали соединения $\mathrm{Pb}_{10-x} \mathrm{La}_{x}\left(\mathrm{GeO}_{4}\right)_{2+x}\left(\mathrm{VO}_{4}\right)_{4-x}(x=0-3)$ с такой же структурой. Подобная замена части свинца на висмут позволила получить апатиты составов $\mathrm{Pb}_{10-x} \mathrm{Bi}_{x}\left(\mathrm{GeO}_{4}\right)_{2+x}\left(\mathrm{VO}_{4}\right)_{4-x}(x=0-3)$ [13]. Замещением свинца на празеодим получены соединения $\mathrm{Pb}_{10-x} \mathrm{Pr}_{x}\left(\mathrm{GeO}_{4}\right)_{2+x}\left(\mathrm{VO}_{4}\right)_{4-x}(x=0-3)$ [6, $10]$. В отличие от хорошо известного гидроксиапатита $[4,14$ - 16] его свинцовые аналоги исследованы недостаточно [6].

Целью настоящей работы является синтез, исследование кристаллической структуры и термодинамических свойств апатитов $\mathrm{Pb}_{10-x} \mathrm{Gd}_{x}\left(\mathrm{GeO}_{4}\right)_{2+x}\left(\mathrm{VO}_{4}\right)_{4-x}(x=0.5,1.0)$.

\section{Экспериментальная часть}

Соединения $\mathrm{Pb}_{10-x} \mathrm{Gd}_{x}\left(\mathrm{GeO}_{4}\right)_{2+x}\left(\mathrm{VO}_{4}\right)_{4-x}(x=0.5,1.0)$ со структурой апатита получали из $\mathrm{PbO}$, $\mathrm{Gd}_{2} \mathrm{O}_{3}$ и $\mathrm{V}_{2} \mathrm{O}_{5}$ - «ос.ч» и $\mathrm{GeO}_{2}-99.999$ \%. Для этого предварительно прокаленные исходные оксиды в стехиометрических количествах перетирали в агатовой ступке. Затем их прессовали в таблетки и обжигали на воздухе при 773, 873, 973 K (по 10 ч при каждой температуре) и $1073 \mathrm{~K}$ (100 ч). Нижний предел температуры твердофазного синтеза связан с наличием в исходной смеси легкоплавких $\mathrm{PbO}$ и $\mathrm{V}_{2} \mathrm{O}_{5}$ и наличием на диаграмме состояния системе $\mathrm{PbO}-\mathrm{V}_{2} \mathrm{O}_{5}$ эвтектики с $T_{\text {пл }}=760 \mathrm{~K}$ [17]. Время и температуры отжига определены на основании предварительных экспериментов. Для увеличения полноты протекания твердофазной реакции через каждые 10 ч проводили перетирание полученных образцов и снова прессовали. Контроль фазового состава осуществляли с использованием рентгеноструктурного анализа. Порошковые рентгенограммы апатитов $\mathrm{Pb}_{10-x} \mathrm{Gd}_{x}\left(\mathrm{GeO}_{4}\right)_{2+x}\left(\mathrm{VO}_{4}\right)_{4-x}(x=0.5,1.0)$ получены при комнатной температуре на дифрактометре Bruker D8 ADVANCE (CuK ${ }_{\alpha}$-излучение) с использованием линейного детекTора VANTEC. Шаг сканирования составлял $0.016^{\circ}$, а время экспозиции на каждый шаг - 2 с. Уточнение Ритвельда проведено в программе TOPAS4.2 [18].

Измерение теплоемкости синтезированных апатитов проводили на термоанализаторе STA 449 C Jupiter (NETZSCH, Германия) методом дифференциальной сканирующей калориметрии. Методика экспериментов подобна описанной в работе [19]. Ошибка экспериментов не превышала $2 \%$.

\section{Результаты и обсуждение}

Все рефлексы были проиндицированы гексагональной ячейкой $\left(P 6_{3} / m\right)$ с параметрами, близкими к $\mathrm{Pb}_{5}\left(\mathrm{GeO}_{4}\right)\left(\mathrm{VO}_{4}\right)_{2}$ [2]. Поэтому эта структура была взята в качестве структурной модели уточнения. Согласно предполагаемой химической формуле, в обе независимые позиции ионов свинца ( $\mathrm{Pb} 1$ и $\mathrm{Pb} 2)$ были помещены ионы $\mathrm{Pb} / \mathrm{Gd}$ с фиксированными значениями заселенностей позиций (рис. 1).

Для единственной позиции $\mathrm{Ge} / \mathrm{V}$ было рассчитано соотношение между Ge: V ионами с учетом химической формулы, и эти заселенности также были фиксированы в ходе уточнения. Тепловые параметры всех атомов уточнены в изотропном приближении. Уточнение шло стабильно и дало низкие величины $R$-факторов (табл. 1, рис. 2). Координаты атомов и основные длины связей представлены в табл. 2 и 3 соответственно.

На рис. 3 показано влияние температуры на значения молярной теплоемкости апатитов $\mathrm{Pb}_{10-x} \mathrm{Gd}_{x}\left(\mathrm{GeO}_{4}\right)_{2+x}\left(\mathrm{VO}_{4}\right)_{4-x}$. Видно, что в интервале от 350 до $1000 \mathrm{~K}$ молярная теплоемкость 


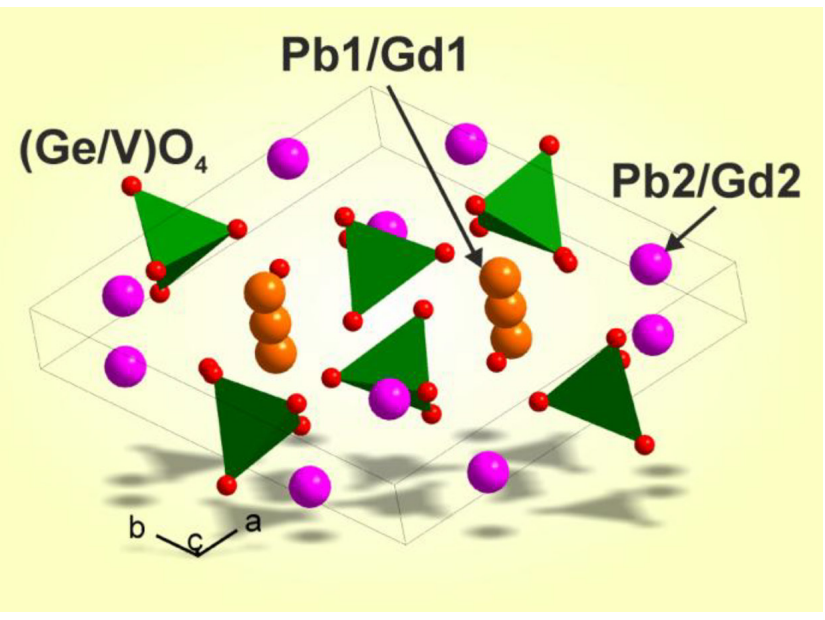

Рис. 1. Кристаллическая структура $\mathrm{Pb}_{10-x} \mathrm{Gd}_{x}\left(\mathrm{GeO}_{4}\right)_{2+x}\left(\mathrm{VO}_{4}\right)_{4-x}$

Fig. 1. Crystal structure of $\mathrm{Pb}_{10-x} \mathrm{Gd}_{x}\left(\mathrm{GeO}_{4}\right)_{2+x}\left(\mathrm{VO}_{4}\right)_{4-x}$

Таблица 1. Основные параметры съемки и уточнения апатитов $\mathrm{Pb}_{10-x} \mathrm{Gd}_{\mathrm{x}}\left(\mathrm{GeO}_{4}\right)_{2+x}\left(\mathrm{VO}_{4}\right)_{4-x}$

Table 1. Main parameters of processing and refinement of the $\mathrm{Pb}_{10-x} \mathrm{Gd}_{\mathrm{x}}\left(\mathrm{GeO}_{4}\right)_{2+x}\left(\mathrm{VO}_{4}\right)_{4-x}$ apathites

\begin{tabular}{|c|c|c|}
\hline $\mathrm{x}$ & 0.5 & 1.0 \\
\hline Пространственная группа & $P 6_{3} / m$ & $P 6_{3} / m$ \\
\hline$a, \AA$ & $10.09373(18)$ & $10.09233(7)$ \\
\hline$c, \AA$ & $7.35697(16)$ & $7.37845(7)$ \\
\hline$V, \AA^{3}$ & $649.13(3)$ & $650.847(11)$ \\
\hline$Z$ & 1 & 1 \\
\hline $2 \theta-$ интервал, $^{\circ}$ & $7.5-120$ & $5.5-120$ \\
\hline$R_{\text {wp }} \%$ & 6.95 & 4.43 \\
\hline$R_{p}, \%$ & 5.15 & 3.81 \\
\hline$R_{\exp }, \%$ & 3.75 & 1.51 \\
\hline$\chi^{2}$ & 1.85 & 1.76 \\
\hline$R_{B}, \%$ & 1.58 & 5.75 \\
\hline
\end{tabular}

Примечание: $a, c$ - параметры ячейки; $V$ - объем ячейки; факторы недостоверности: $R_{w p}-$ весовой профильный, $R_{p}-$ профильный, $R_{\exp }$ - ожидаемый; $R_{B}$ - интегральный; $\chi^{2}$ - качество подгонки.

закономерно увеличивается, а на зависимости нет различного рода экстремумов. Это позволяет заключить, что в этой области температур у исследованных апатитов не полиморфные превращения. Обращает на себя внимание близость значений теплоемкости при $x=0,5$ и 1,0. Экспериментальные данные по теплоемкости в исследованном интервале температур хорошо описываются классическим уравнением Майера - Келли

$$
C_{p}=a+b T-c T^{-2}
$$

которое имеет следующий вид для $\mathrm{Pb}_{10-x} \mathrm{Gd}_{x}\left(\mathrm{GeO}_{4}\right)_{2+x}\left(\mathrm{VO}_{4}\right)_{4-x}$ (Дж/(моль $\left.\mathrm{K}\right)$ ): 

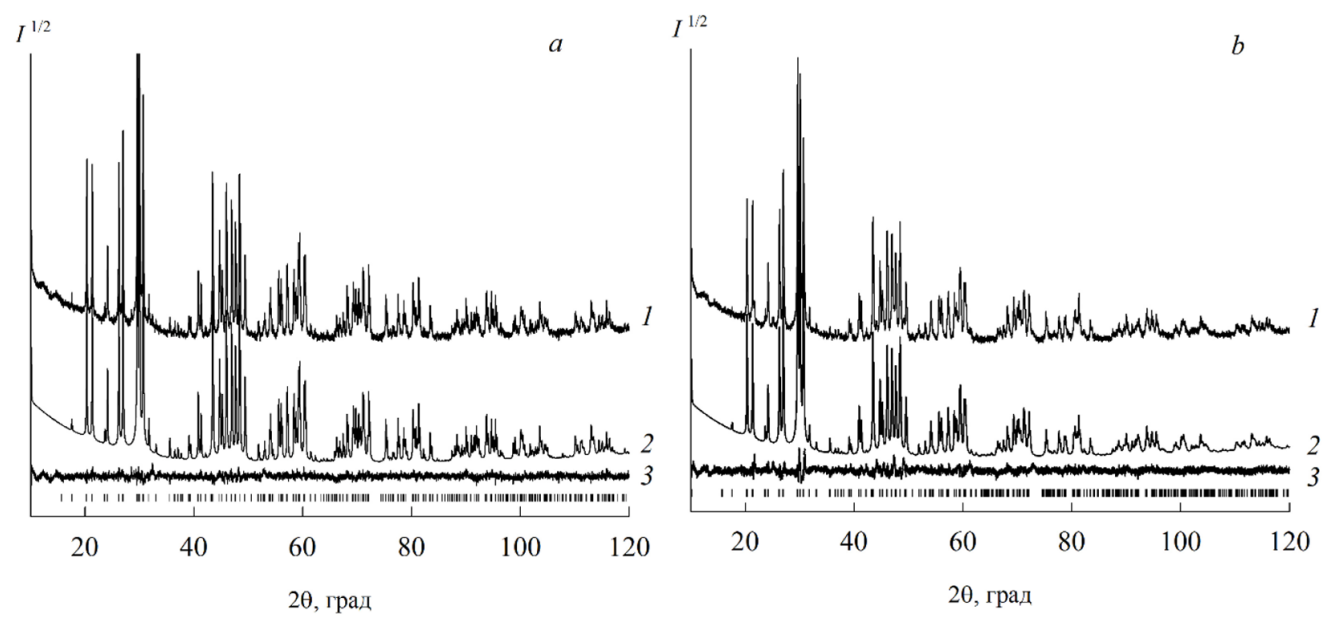

Рис. 2. Разностные рентгенограммы уточнения Ритвельда $\mathrm{Pb}_{10-x} \mathrm{Gd}_{x}\left(\mathrm{GeO}_{4}\right)_{2+x}\left(\mathrm{VO}_{4}\right)_{4-x}: x=1(a) ; 0.5(b)$. Экспериментальный (1), расчетный (2) и разностный (3) профили рентгенограмм; штрихи указывают расчетные положение рефлексов

Fig. 2. Difference Rietveld plot of $\mathrm{Pb}_{10-x} \mathrm{Gd}_{x}\left(\mathrm{GeO}_{4}\right)_{2+x}\left(\mathrm{VO}_{4}\right)_{4-x}: x=0.5(a) ; 1(b)$. Experimental, (2) calculated, and (3) difference profiles, marks show the calculated reflection positions

Таблица2. Атомныекоординаты и изотропныетепловыепараметры $\left(\AA^{2}\right)$ апатитов $\mathrm{Pb}_{10-x} \mathrm{Gd}_{x}\left(\mathrm{GeO}_{4}\right)_{2+x}\left(\mathrm{VO}_{4}\right)_{4-x}$ Table 2. Fractional atomic coordinates and isotropic displacement parameters $\left(\AA^{2}\right)$ of $\mathrm{Pb}_{10-\mathrm{x}} \mathrm{Gd}_{\mathrm{x}}\left(\mathrm{GeO}_{4}\right)_{2+\mathrm{x}}\left(\mathrm{VO}_{4}\right)_{4-\mathrm{x}}$ apathites

\begin{tabular}{|c|c|c|c|c|c|}
\hline Атом & $\mathrm{x}$ & $\mathrm{y}$ & $\mathrm{z}$ & $\mathrm{B}_{\text {iso }}$ & Occ. \\
\hline \multicolumn{6}{|c|}{$x=0.5$} \\
\hline $\mathrm{Pb} 1$ & $1 / 3$ & $2 / 3$ & $0.0077(7)$ & $1.45(10)$ & 0.95 \\
\hline Gd1 & $1 / 3$ & $2 / 3$ & $0.0077(7)$ & $1.45(10$ & 0.05 \\
\hline $\mathrm{Pb} 2$ & $0.2558(2)$ & $0.0055(4)$ & $1 / 4$ & $1.43(10$ & 0.95 \\
\hline $\mathrm{Gd} 2$ & $0.2558(2)$ & $0.0055(4)$ & $1 / 4$ & $1.43(10$ & 0.05 \\
\hline $\mathrm{Ge}$ & $0.4039(6)$ & 0.3827 & $1 / 4$ & $0.30(16)$ & $5 / 12$ \\
\hline $\mathrm{V}$ & $0.4039(6)$ & $0.3827(7)$ & $1 / 4$ & $0.30(16)$ & $7 / 12$ \\
\hline $\mathrm{O} 1$ & $0.305(3)$ & $0.465(3)$ & $1 / 4$ & $3 / 5(5)$ & 1 \\
\hline $\mathrm{O} 2$ & $0.593(3)$ & $0.507(2)$ & $1 / 4$ & $3 / 5(5)$ & 1 \\
\hline $\mathrm{O} 3$ & $0.3539(17)$ & $0.2655(18)$ & $0.073(2)$ & $3 / 5(5)$ & 1 \\
\hline \multicolumn{6}{|c|}{$x=1.0$} \\
\hline $\mathrm{Pb} 1$ & $1 / 3$ & $2 / 3$ & $0.0061(5)$ & $1.02(6)$ & 0.9 \\
\hline $\mathrm{Gd} 2$ & $1 / 3$ & $2 / 3$ & $0.0061(5)$ & $1.02(6)$ & 0.1 \\
\hline $\mathrm{Pb} 2$ & $0.25399(16)$ & $0.0032(3)$ & $1 / 4$ & $1.02(6)$ & 0.9 \\
\hline $\mathrm{Gd} 2$ & $0.25399(16)$ & $0.0032(3)$ & $1 / 4$ & $1.02(6)$ & 0.1 \\
\hline $\mathrm{Ge}$ & $0.4000(5)$ & $0.3840(5)$ & $1 / 4$ & $0.64(10)$ & 0.5 \\
\hline $\mathrm{V}$ & $0.4000(5)$ & $0.3840(5)$ & $1 / 4$ & $0.64(10)$ & 0.5 \\
\hline $\mathrm{O} 1$ & $0.2994(18)$ & $0.4767(19)$ & $1 / 4$ & $2.5(3)$ & 1 \\
\hline $\mathrm{O} 2$ & $0.5954(17)$ & $0.5023(17)$ & $1 / 4$ & $2.5(3)$ & 1 \\
\hline $\mathrm{O} 3$ & $0.3541(12)$ & $0.2589(12)$ & $0.0678(13)$ & $2.5(3)$ & 1 \\
\hline
\end{tabular}


Таблица 3. Основные длины связей $(\AA) \mathrm{Pb}_{10-x} \mathrm{Gd}_{x}\left(\mathrm{GeO}_{4}\right)_{2+x}\left(\mathrm{VO}_{4}\right)_{4-x}$

Table 3. Main bond lengths $(\AA)$ of $\mathrm{Pb}_{10-x} \mathrm{Gd}_{x}\left(\mathrm{GeO}_{4}\right)_{2+x}\left(\mathrm{VO}_{4}\right)_{4-x}$

\begin{tabular}{|c|c|c|c|}
\hline \multicolumn{4}{|c|}{$x=0.5$} \\
\hline$(\mathrm{Pbl} / \mathrm{Gd} 1)-\mathrm{O} 1$ & $2.612(19)$ & $(\mathrm{Ge} / \mathrm{V})-\mathrm{O} 1$ & $1.586(18)$ \\
\hline$(\mathrm{Pb} 1 / \mathrm{Gd} 1)-\mathrm{O} 2^{\mathrm{i}}$ & $2.920(16)$ & $\mathrm{Ge} / \mathrm{V})-\mathrm{O} 2$ & $1.68(2)$ \\
\hline$(\mathrm{Pb} 1 / \mathrm{Gd} 1)-\mathrm{O}^{\mathrm{ii}}$ & $2.937(15)$ & $\mathrm{Ge} / \mathrm{V})-\mathrm{O} 3$ & $1.659(16)$ \\
\hline$(\mathrm{Pb} 2 / \mathrm{Gd} 2)-\mathrm{O} 1^{\mathrm{iii}}$ & $2.78(3)$ & & \\
\hline$(\mathrm{Pb} 2 / \mathrm{Gd} 2)-\mathrm{O} 2^{\mathrm{iv}}$ & 2.11(2) & & \\
\hline$(\mathrm{Pb} 2 / \mathrm{Gd} 2)-\mathrm{O} 3$ & $2.639(16)$ & & \\
\hline$(\mathrm{Pb} / \mathrm{Gd} 2)-\mathrm{O}^{\mathrm{v}}$ & $2.578(15)$ & & \\
\hline \multicolumn{4}{|c|}{$x=1.0$} \\
\hline$(\mathrm{Pbl} / \mathrm{Gd} 1)-\mathrm{O} 1$ & $2.525(11)$ & $(\mathrm{Ge} / \mathrm{V})-\mathrm{O} 1$ & $1.690(11)$ \\
\hline$(\mathrm{Pb} 1 / \mathrm{Gd} 1)-\mathrm{O} 2^{\mathrm{i}}$ & $2.867(11)$ & $\mathrm{Ge} / \mathrm{V})-\mathrm{O} 2$ & $1.720(12)$ \\
\hline$(\mathrm{Pb} 1 / \mathrm{Gd} 1)-\mathrm{O} 3^{\mathrm{ii}}$ & $2.906(10)$ & $\mathrm{Ge} / \mathrm{V})-\mathrm{O} 3$ & $1.741(10)$ \\
\hline$(\mathrm{Pb} 2 / \mathrm{Gd} 2)-\mathrm{O} 1^{\mathrm{iii}}$ & $2.750(16)$ & & \\
\hline$(\mathrm{Pb} 2 / \mathrm{Gd} 2)-\mathrm{O} 2^{\mathrm{iv}}$ & $2.154(15)$ & & \\
\hline$(\mathrm{Pb} 2 / \mathrm{Gd} 2)-\mathrm{O} 3$ & $2.623(10)$ & & \\
\hline$(\mathrm{Pb} / \mathrm{Gd} 2)-\mathrm{O}^{\mathrm{v}}$ & $2.557(10)$ & & \\
\hline
\end{tabular}

Примечание. Элементы симметрии: (i) $-x+1,-y+1, z-1 / 2$; (ii) y, -x+y+1, -z; (iii) $-x+y,-x,-z+1 / 2$; (iv) $-y+1, x-y,-z+1 / 2$; (v) $y$, $-x+y,-z$.

$$
\begin{aligned}
& x=0,5 \\
& C p=(889.4 \pm 5.2)+(202.7 \pm 5.40) 10^{-3} T-(72.12 \pm 5.60) 10^{5} T^{-2}, \\
& x=1,0 \\
& C p=(865.9 \pm 4.2)+(237.9 \pm 4.40) 10^{-3} T-(31.85 \pm 4.57) 10^{5} T^{-2} .
\end{aligned}
$$

Для уравнений (2) и (3) коэффициенты корреляции равны 0.9980 и 0.9987 , а максимальное отклонение экспериментальных точек от сглаживающих кривых - 3.3 и $2.6 \%$ соответственно. С использованием уравнений (2) и (3) по известным термодинамическим соотношениям рассчитаны термодинамические функции исследованных апатитов. В качестве примера в табл. 4 приведены данные для $\mathrm{Pb}_{9} \mathrm{Gd}\left(\mathrm{GeO}_{4}\right)_{3}\left(\mathrm{VO}_{4}\right)_{3}$.

Сравнить полученные нами результаты по высокотемпературной теплоемкости апатитов $\mathrm{Pb}_{10-x} \mathrm{Gd}_{x}\left(\mathrm{GeO}_{4}\right)_{2+x}\left(\mathrm{VO}_{4}\right)_{4-x}$ с данными других авторов не представлялось возможным вследствие их отсутствия. Такое сравнение провели с рассчитанными величинами по различным модельным представлениям: аддитивным методом Неймана-Коппа (НК) [20], инткрементным методом Кумока (ИМК) [21], Келлога (Кел) [22] и групповых вкладов (ГВ) [22]. Эти данные приведены в табл. 5. Из их анализа следует, что лучшее согласие с экспериментальными значениями метод Келлога.

Для расчета температурной зависимости теплоемкости твердых тел используют различные модельные представления [21-24]. Их использование вызывает необходимость иметь дополнительные сведения о температуре плавления, характеристической температуре Де- 


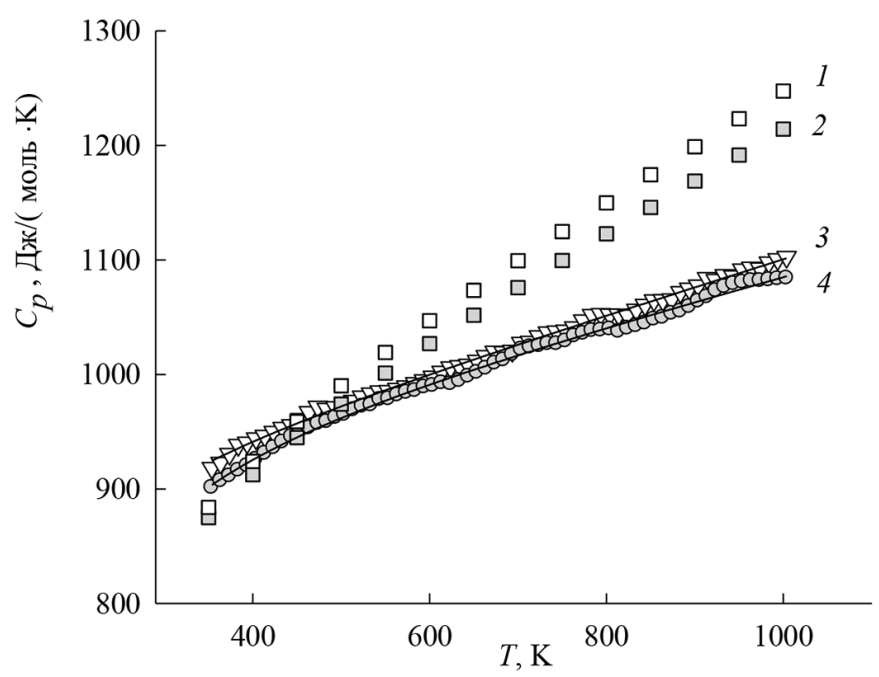

Рис. 3. Влияние температуры на молярную теплоемкость $\mathrm{Pb}_{10-x} \mathrm{Gd}_{x}\left(\mathrm{GeO}_{4}\right)_{2+x}\left(\mathrm{VO}_{4}\right)_{4-x} x=1.0(1,3)$ и $0,5(2$, 4): 1, 2 -расчет методом групповых вкладов; 3, 4-экспериментальные данные

Fig. 3. Effect of temperature on the specific heat of $\mathrm{Pb}_{10-x} \mathrm{Gd}_{x}\left(\mathrm{GeO}_{4}\right)_{2+x}\left(\mathrm{VO}_{4}\right)_{4-x}$ with $x=1.0(1,3)$ and $0,5(2,4)$ : $(1,2)$ group contribution calculation; $(3,4)$ experimental data

Таблица 4. Термодинамические свойства $\mathrm{Pb}_{9} \mathrm{Gd}\left(\mathrm{GeO}_{4}\right)_{3}\left(\mathrm{VO}_{4}\right)_{3}$

Table 4. Thermodynamic properties of the $\mathrm{Pb}_{9} \mathrm{Gd}\left(\mathrm{GeO}_{4}\right)_{3}\left(\mathrm{VO}_{4}\right)_{3}$

\begin{tabular}{|c|c|c|c|c|}
\hline$T, \mathrm{~K}$ & $\begin{array}{c}C_{P}, \\
\text { Дж·моль }{ }^{-1} \cdot \mathrm{K}^{-1}\end{array}$ & $\begin{array}{c}H^{\circ}(T)-H^{\circ}(350 \mathrm{~K}), \\
\text { кДжмоль }\end{array}$ & $\begin{array}{c}S^{\mathrm{o}}(T)-S^{\mathrm{o}}(350 \mathrm{~K}), \\
\text { Дж·моль }{ }^{-1} \cdot \mathrm{K}^{-1}\end{array}$ & $\begin{array}{c}-\Delta G / T^{*}, \\
\text { Дж моль-1 } \mathrm{K}^{-1}\end{array}$ \\
\hline 350 & 923.1 & - & - & - \\
\hline 400 & 941.1 & 46.61 & 124.5 & 7.93 \\
\hline 450 & 957.2 & 94.08 & 236.3 & 27.19 \\
\hline 500 & 972.1 & 142.3 & 337.9 & 53.25 \\
\hline 550 & 986.2 & 191.3 & 431.2 & 83.43 \\
\hline 600 & 999.7 & 240.9 & 517.6 & 116.1 \\
\hline 650 & 1013 & 291.2 & 598.1 & 150.1 \\
\hline 700 & 1025 & 342.2 & 673.7 & 184.8 \\
\hline 750 & 1038 & 393.8 & 744.9 & 219.8 \\
\hline 800 & 1051 & 446.1 & 812.3 & 254.7 \\
\hline 850 & 1064 & 498.9 & 876.4 & 289.4 \\
\hline 900 & 1076 & 552.4 & 937.6 & 323.7 \\
\hline 950 & 1088 & 606.5 & 996.1 & 357.6 \\
\hline 1000 & 1101 & 661.3 & 1052 & 390.9 \\
\hline
\end{tabular}

Примечание* $-\Delta G / T^{*}=\left[H^{\circ}(T)-\mathrm{H}^{\circ}(350 \mathrm{~K})\right] / T-\left[\mathrm{S}^{\circ}(T)-\mathrm{S}^{\circ}(350 \mathrm{~K})\right]$.

бая и др. Такие данные для апатитов $\mathrm{Pb}_{10-x} \mathrm{R}_{x}\left(\mathrm{GeO}_{4}\right)_{2+x}\left(\mathrm{VO}_{4}\right)_{4-x}(\mathrm{R}-$ редкоземельный элемент) в литературе отсутствуют. Метод групповых вкладов [23] позволяет рассчитывать $C p=f(T)$ без каких-либо дополнительных сведений о сложных оксидных соединениях. Нами проведен расчет температурных зависимостей теплоемкости исследованных апатитов (рис. 3 , кривые 1 и 2). Если при низких температурах экспериментальные и рассчитанные значения теплоемкостей близки, то с ростом температуры наблюдается их различие (чем выше температура, тем больше разница). Следует отметить, что подобное наблюдали и авторы работы [24], и ав- 
Таблица 5. Сравнение экспериментальных значений молярной теплоемкости $\mathrm{Pb}_{10-x} \mathrm{Gd}_{x}\left(\mathrm{GeO}_{4}\right)_{2+x}\left(\mathrm{VO}_{4}\right)_{4-x}$ с рассчитанными величинами по различным модельным представлениям $\left(C_{p 298}\right.$, Дж/(моль:К)

Table 5. Comparison of experimental values of moly thermal capacity $\mathrm{Pb}_{10-x} \mathrm{Gd}_{x}\left(\mathrm{GeO}_{4}\right)_{2+x}\left(\mathrm{VO}_{4}\right)_{4-x}$ with calculated values on different model views $C_{p 298}(\mathrm{~J} /(\mathrm{mol} \mathrm{K}))$

\begin{tabular}{|c|c|c|c|c|c|c|c|c|c|}
\hline$x$ & $C_{p}$, экс & НК & $\Delta, \%$ & ИМК & $\Delta, \%$ & Кел & $\Delta, \%$ & $\Gamma$ Г & $\Delta, \%$ \\
\hline$x=0.5$ & 868.6 & 811.3 & -6.6 & 827.8 & -4.7 & 833.1 & -4.1 & 826.3 & -4.9 \\
\hline$x=1.0$ & 900.9 & 813.6 & -9.6 & 828.2 & -8.1 & 835.8 & -7.2 & 831.8 & -7.7 \\
\hline
\end{tabular}

торы данного метода [25]. Это позволяет считать, что метод групповых вкладов не является универсальным.

\section{Список литературы / References}

1. Игнатов А. В., Дидоренко Е. Г., Чебышев К. А. и др. Изоморфное замещение свинца самарием в структуре $\mathrm{Ca}_{2} \mathrm{~Pb}_{2} \mathrm{Na}_{2}\left(\mathrm{PO}_{4}\right)_{6}$. Вестн. Донеикого наи. ун-та. Сер. А. Естественные науки. 2021. № 1. C. 62-69. [Ignatov A. V., Didorenko E. G., Chebyshev K. A. et al. Isomorphous substitutions of lead on samarium in the structure $\mathrm{Ca}_{2} \mathrm{~Pb}_{2} \mathrm{Na}_{2}\left(\mathrm{PO}_{4}\right)_{6}$. Vestn. Donetsk. Nat. Univ. Ser. A. 2021. № 1. P. 62-69. (In Russ.)].

2. Иванов С. А. Уточнение кристаллической структуры $\mathrm{Pb}_{5}\left(\mathrm{GeO}_{4}\right)\left(\mathrm{VO}_{4}\right)_{2}$ по профилю порошковых рентгенограмм. Журн. структурн. химии. 1990. T. 31 (4). C. 80-84. [Ivanov S. A. Crystal structure refinement of $\mathrm{Pb}_{5}\left(\mathrm{GeO}_{4}\right)\left(\mathrm{VO}_{4}\right)_{2}$ using X-ray powder diffraction pear profiles. Zh. Strukt. Khim. 1990. V. 31(4). P. 80-84. (In Russ.)].

3. Vasin A. A., Zuev M. G., Popov I.D. et al. High-pressure $\mathrm{Eu}^{3+} \rightarrow \mathrm{Eu}^{2+}$ reduction in a matrix with the structure of apatite silicate Russ. J. Phys. Chem. A. 2020. T. 94. № 12. P. 2467-2473.

4. Pokhrel S. Hydroxyapatite: Preparation, Properties and Its Biomedical Applications. Adv. Chem. Eng. Sci. 2018. V. 8. P. 225-240.

5. Bulanov E. N., Korshak K. S., Lelet M. I. et al. Bi-apatite: synthesis, crystal structure and lowtemperature heat capacity. J. Chem. Thermodynamics. 2018. V. 1. 124. P. 74-78.

6. Yablochkova N. V. Synthesis of $\mathrm{Pb}_{8} \mathrm{Pr}_{2}\left(\mathrm{GeO}_{4}\right)_{4}\left(\mathrm{VO}_{4}\right)_{2}$ and refinement of its crystal structure. J. Inorg. Chem. 2013. V1. 58 (7). P. 769-772.

7. Savankova T.M., Akselrud L. G., Ardanova L. I. et al. Synthesis, crystal structure refinement, and electrical conductivity of $\mathrm{Pb}_{(8-\mathrm{x})} \mathrm{Na}_{2} \mathrm{Sm}_{\mathrm{x}}\left(\mathrm{VO}_{4}\right)_{6} \mathrm{O}_{(\mathrm{x} / 2)}$. J. Chem. 2014. P. 1-7.

8. Bulanov E. N., Petrov S. S., Xu Z. et al. Synthesis and crystal structure pf some Ba-apatite. Rus. J. Inorg. Chem. 2021. V. 66. № 4. C. 455-459.

9. Zhang F.X., Lang M., Zhang J.M. et al. Phase transition and abnormal compressibility of lanthanide silicate with the apatite structure. Phys. Rev. B. 2012. V. 85. P. 214116-1-214116-6.

10. Denisova L. T., Kargin Yu.F., Golubeva E. O. et al. Heat Capacity of $\mathrm{Pb}_{10-\mathrm{x}} \mathrm{Pr}_{\mathrm{x}}\left(\mathrm{GeO}_{4}\right)_{2+\mathrm{x}}\left(\mathrm{VO}_{4}\right)_{4-\mathrm{x}}$ $(\mathrm{x}=0,1,2,3)$ apatites in the range 350-1050 K. Inorg. Mater. 2020. V. 56 (10). P. 1027-1032.

11. Игнатов А.В., Саванкова Т.М., Дидоренко Е.Г. и др. Изоморфное замещение в системе $\mathrm{Pb}_{(8-\mathrm{x})} \mathrm{Gd}_{\mathrm{x}} \mathrm{Na}_{2}\left(\mathrm{VO}_{4}\right)_{6} \mathrm{O}_{(\mathrm{x} / 2)}$. Вестн. Донеикого наи. ун-та. Сер. А. 2014. № 1. С. 152-156. [Ignatov A. V., Savankova T.M., Didorenko E. G. et al. Isomorphous substitutions in the $\mathrm{Pb}_{(8-\mathrm{x})} \mathrm{Gd}_{\mathrm{x}}$ $\mathrm{Na}_{2}\left(\mathrm{VO}_{4}\right)_{6} \mathrm{O}_{(\mathrm{x} / 2)}$ system. Vestn. Donetsk. Nat. Univ. Ser. A. 2014. № 1. P. 152-156. (In Russ.)]. 
12.Zhuravlev V.D., Velikodny Yu. A. Leadlanthanum and strontium lanthanumgermanovanadates with apatite and oxyapatite structures. J. Inorg. Chem. 2009. Vol. 54 (10). P. 1551-1552.

13. Denisova L. T., Molokeev M. S., Denisov V.M. et al. Synthesis, structure, and thermophysical properties of $\mathrm{Pb}_{10-\mathrm{x}} \mathrm{Bi}_{\mathrm{x}}\left(\mathrm{GeO}_{4}\right)_{2+\mathrm{x}}\left(\mathrm{VO}_{4}\right)_{4-\mathrm{x}}(x=0-3)$ in the temperature range of 350-950 K. Phys. Solid State. 2020. V. 62 (11). P. 2045-2051.

14. Krut'ko V.K., Kulak A.I., Musskaya O.N. Thermal transformation of composites based on hydroxyapatite and zirconia. Inorg. Mater. 2017. V. 53. № 4. P. 429-436.

15. Каржавин В.К. Термодинамические величины химических элементов и соединений. Примеры их практического применения. Апатиты: Изд-во Кольск. научн. центра РАН, 2011. 160 c. [Karzhavin V.K. Thermodynamic parameters of chemical elements and compounds. Apatity: Kol'skii Nauchnyi Tsentr Ross. Akad. Nauk. 2011.160 p. (In Russ)].

16. Chaikina M.V., Bulina N.V., Prosanov I. Y. et al. Structure formation of zinc-substituted hydroxyapatite during mechanochemical synthesis. Inorg. Mater. 2020. V. 56. № 4. P. 402-408.

17. Xie W., Wang N., Qiao Zh., Cao Zh. Thermodynamic assessment of the $\mathrm{PbO}-\mathrm{V}_{2} \mathrm{O}_{5}$ system. CALPHAD: Computer Coupling of Phase Diagrams and Thermochemistry. 2016. V. 55. P. 41-46.

18. Bruker AXS TOPAS V4: General profile and structure analysis software for powder diffraction data. - User's Manual. Bruker AXS, Karlsruhe, Germany, 2008.

19. Denisova L. T., Irtyugo L. A., Kargin Yu.F., Beletskii V. V., Denisov V. M. High- temperature heat capacity and thermodynamic properties of $\mathrm{Tb}_{2} \mathrm{Sn}_{2} \mathrm{O}_{7}$. Inor. Mater. 2017 . V. 53 (1). P. 93-95.

20. Laitner J., Chuchvalec P., Sedmidubský D. et al. Estimation of heat capacities of solid mixed oxides. Thermochim. Acta. 2003. V. 395. P. 27-46.

21. Кумок В.Н. Проблема согласования методов оценки термодинамических характеристик. Прямые и обратные задачи химической термодинамики. Новосибирск: Наука, 1987. C. 108-123. [Pryamye i obratnye zadachi khimicheskoi termodinamiki (Direct and inverse problems of chemical thermodynamics). Novosibirsk: Nauka, 1987. P. 108-123. (In Russ)].

22. Кубашевский О., Олкокк С.Б. Металлургическая химия. М.: Металлургия, 1982. 392 с. [Kubaschewski O., Alcock C.B. Metallurgical thermochemistry. Pergamon Press Lid. 1982. 392 p. (in Russ)].

23. Mostafa A. T.M.G., Eakman J.M., Montoya M.M. et al. Prediction of heat capacities of solid inorganic salts from group contributions. Ind. Eng. Chem. Res. 1996. V. 35. P. 343-348.

24. Морачевский А.Г., Сладков И.Б., Фирсова Е.Г. Термодинамические расчеты в химии и металлургии. СПб.: Лань, 2018. 208 с. [Morachevskii A. G., Sladkov I. G., and Firsova E. G. Thermodynamic Calculations in Chemistry and Metallurgy. Lan'.: St. Petersburg, 2018. 208 p.) (in Russ].

25. Leitner J., Sedmidubský D., Chuchvalec P. Prediction of Heat Capacities of Solid Binary Oxides from Group Contribution Method. Ceramics-Silikáty. 2002. V. 46. № 1. P. 29-32. 